\title{
Usefulness of cyclic thermal therapy and red blood cell scintigraphy in patients with chemotherapy-induced peripheral neuropathy
}

\author{
Minjoo Kim ${ }^{1}$, Eun-Mi Kim ${ }^{1}$, Phil-Sun Oh${ }^{1}$, Seok Tae Lim ${ }^{1}$, Myung-Hee Sohn ${ }^{1}$, Eun-Kee Song ${ }^{2}$, Keon Uk Park ${ }^{3}$, \\ Jin Young Kim ${ }^{3}$, Kyoung Sook Won ${ }^{4}$, and Hwan-Jeong Jeong ${ }^{1}$
}

'Department of Nuclear Medicine, Molecular Imaging \& Therapeutic Medicine Research Center, Research Institute of Clinical Medicine, Biomedical Research Institute, Jeonbuk National University Medical School and Hospital, Jeonju, Korea

${ }^{2}$ Division of Medical Oncology \& Hematology, Department of Internal Medicine, Jeonbuk National University Medical School and Hospital, Jeonju, Korea

${ }^{3}$ Department of Hematology/Oncology, Keimyung University Dongsan Hospital, Daegu, Korea

${ }^{4}$ Department of Nuclear Medicine, Keimyung University Dongsan Hospital, Daegu, Korea

Received March 5, 2021

Revised June 1, 2021

Accepted June 7, 2021

Handling Editor: Jae Hun Kim

\section{Correspondence}

Hwan-Jeong Jeong

Department of Nuclear Medicine,

Molecular Imaging \& Therapeutic

Medicine Research Center, Research

Institute of Clinical Medicine, Biomedical

Research Institute, Jeonbuk National

University Medical School and Hospital,

20, Geonji-ro, Jeonju 54907, Korea

Tel: +82-63-250-1674

Fax: +82-63-255-1172

E-mail: jayjeong@jbnu.ac.kr
Background: Pharmacological and non-pharmacological therapies have been used to treat patients with chemotherapy-induced peripheral neuropathy (CIPN). However, the effect of therapies in cancer patients has yet to be investigated comprehensively. We hypothesized that cyclic thermal therapy would improve blood flow and microcirculation and improve the symptoms driven by CIPN.

Methods: The criteria of assessment were blood volume in region of interest (ROI) in the images, and European Organization for Research and Treatment of CancerQuality of Life Questionnaire-Chemotherapy-Induced Peripheral Neuropathy 20 questionnaire scores. The blood volume was quantified by using red blood cell (RBC) scintigraphy. All patients were treated 10 times during 10 days. The thermal stimulations, between $15^{\circ}$ and $41^{\circ}$, were repeatedly delivered to the patient's hands. Results: The total score of the questionnaires, the score of questions related to the upper limbs, the score of questions closely related to the upper limbs, and the score excluding the upper limbs questions was decreased. The blood volume was decreased, and the variance of blood volume was decreased. During cooling stimulation, the blood volume was decreased, and its variance was decreased. During warming stimulation, the blood volume was decreased, and its variance was decreased.

Conclusions: We suggest that cyclic thermal therapy is useful to alleviate CIPN symptoms by blood circulation improvement. RBC scintigraphy can provide the quantitative information on blood volume under certain conditions such as stress, as well as rest, in peripheral tissue.

Key Words: Antineoplastic Agents; Blood Volume; Drug-Related Side Effects and Adverse Reactions; Drug Therapy; Microcirculation; Neuralgia; Peripheral Nervous System Diseases; Polyneuropathies; Radionuclide Imaging; Scintigraphy; Small Fiber Neuropathy. (c) This is an open-access article distributed under the terms of the Creative Commons Attribution Non-Commercial License (http://creativecommons.org/licenses/by-nc/4.0/), which permits unrestricted non-commercial use, distribution, and reproduction in any medium, provided the original work is properly cited.

(c) The Korean Pain Society, 2021
Author contributions: Minjoo Kim: Writing/manuscript preparation; EunMi Kim: Investigation; Phil-Sun Oh: Investigation; Seok Tae Lim: Writing/ manuscript preparation; Myung-Hee Sohn: Writing/manuscript preparation; Eun-Kee Song: Data curation; Keon Uk Park: Data curation; Jin Young Kim: Data curation; Kyoung Sook Won: Writing/manuscript preparation; Hwan-Jeong Jeong: Project administration. 


\section{INTRODUCTION}

Chemotherapy is one of the main treatments for cancer, but it also affects normal cells along with the cancer cells, causing various side effects. Disorders of the nervous system are the second most common side-effect [1]. Chemotherapy-induced peripheral neuropathy (CIPN) is a disorder associated with exercise, sensation, and autonomic nerves, caused by damage to the peripheral nerve after anti-cancer drug treatment, which eventually leads to various symptoms such as muscle weakness, decreased sensation, and pain, disrupting daily life [2-4]. The pain associated with CIPN is distinguished from cancer pain caused by direct invasion of nerve tissues and pressure on the nerves or passage through the bone. It occurs in approximately $40 \%$ of patients undergoing complex chemotherapy. Anti-cancer drugs associated with CIPN include platinum, vinca alkaloids, bortezomib, and taxanes [5].

Pharmacological and non-pharmacological therapies have been used to treat patients with CIPN. Pharmacological therapy consists of calcium and magnesium, vitamin E, glutathione, amifostin, gabapentin, carbamazepine, amythriptyline, and narcotic painkillers. Non-pharmacological therapies include transcutaneous nerve stimulation (TENS), relaxation therapy, exercise, physical therapy, and massage; however, the effect of non-pharmacological therapies in cancer patients has yet to be investigated comprehensively because of its limited use [6,7].

The direct cause of peripheral neuropathy is attributed to peripheral nerve injury and micro-sized blood vessels triggered by the use of neurotoxic anti-cancer drugs. The pathophysiological mechanism involves a direct damage to the peripheral nerve, induced by exposure to toxic anticancer drugs. It is also mediated by nerve damage induced by damage to the associated peripheral micro-sized blood vessels and decreased microvascular reactivity, due to defective endothelial cells [8]. The symptoms include pain and edema. Thermal therapy is used to alleviate pain and edema, and entails stimulation of blood flow with hot temperature for symptom relief $[9,10]$.

Microvascular reactivity is elicited by sustained and consistent blood volume under stress in specific regions of the body. Cold temperature induces vasoconstriction of skin and subcutaneous regions, whereas hot conditions promote vasodilation. Under these external thermal conditions, blood distribution in the body is coordinated secondarily with the maintenance of individual body function. Recovery from endothelial damage and impaired vascular reactivity is attributed to repeated and consecutive vasoconstriction and vasodilation in the micro-vessels, because vessels may undergo stress preconditioning due to change of blood flow mediated via vasoconstrictive and vasodilatory effects [11-13]. Physical exercise is known to prevent the progression of vascular disease [14]. We expected that vasoconstriction and vasodilation via bidirectional thermal stimulation could maintain or recover vascular reactivity by contraction and relaxation of peripheral blood vessels in the peripheral tissues.

Red blood cell (RBC) scintigraphy is used for the quantitative assessment of blood distribution by labeling RBCs with radioactive isotopes. It is used to clinically perform assessments of vascular reactivity and diagnose Raynaud syndrome, which is caused by desecrated vascular reactivity. Both qualitative and quantitative assessments are possible in proceeding scintigraphical analysis [15-19].

In this study, we acted two new theories. One was that stress RBC scintigraphy was performed and the data was quantitatively analyzed and evaluated under the assumption that one of the mechanisms behind the symptoms of CIPN patients could be similar to Raynaud's syndrome. The other was proposing thermal therapy from the perspective of peripheral vascular or tissue exercise as a way to improve the symptoms of CIPN patients, and the introduction of bidirectional thermal therapy rather than cold or hot unidirectional thermal therapy. In addition, the research conducted a subjective assessment of the degree and characteristics of CIPN symptoms through questionnaire testing. We assumed that CIPN symptoms would be related to vascular reactivity and confirmed this correlation through comparative evaluation of questionnaire tests and RBC scintigraphy results.

To explain in detail, the patients' hands were exposed to cycles of cooling and warming stimuli using a cyclic therapeutic device during the RBC scintigraphy. Cyclic stimuli induce vasodilation and vasoconstriction consecutively. We hypothesized that these cyclic stimuli enhance the microcirculation in the peripheral tissue. We defined cyclic cooling and warming therapy by setting the highest and the lowest temperatures, and employing exposure to repetitive stimuli to raise and lower the target temperatures. Fingertips carry a higher number of capillaries than other areas of the fingers, and experience greater variations in temperature. Most of the actual symptoms of CIPN patients, such as tingling sensations and pain, are felt at the fingertips. Therefore, the vascular reactivity to thermal stimuli can be measured via analysis of the fingertips, using RBC scintigraphy.

This study was conducted to evaluate the usefulness of cyclic thermal therapy and RBC scintigraphy in patients with CIPN in investigator-initiated clinical trials (IIT). In this study, we measured the symptom improvement following therapy and observed the changes in the blood volume using RBC scintigraphy of the fingertips. 


\section{MATERIALS AND METHODS}

\section{Ethics approval}

All procedures performed in studies involving human participants were in accordance with the ethical standards of the institutional and/or national research committee, and with the 1964 Helsinki declaration and its later amendments or comparable ethical standards.

\section{Informed consent}

Informed consent approved by the institution was obtained for this study. The informed consent number is CBNUHLNMD_IRB_2017P001 (ver.2.4).

\section{Study design}

This multi-center study was conducted to investigate the benefit of cyclic thermal therapy in patients treated with CIPN from October 2017 to June 2019 at the Departments of Hemato-oncology and Nuclear Medicine of two university hospitals. The research protocol was approved by the Korean Ministry of Food and Drug Safety (No.788), Jeonbuk National University Hospital Institutional Review Board (IRB) and Keimyeong University Dongsan Hospital IRB (approval no. CUH201708022, and DSMC201811005, respectively). The study was performed in accordance with relevant guidelines/regulations, and informed consent was obtained from all participants and/or their legal guardians. This study was designed as a non-randomized, single intervention.

The subjects were screened when they visited the hospital a total of 12 times. During visit 1 (day 0), the European Organization for Research and Treatment of Cancer-Quality of Life Questionnaire-Chemotherapy-Induced Peripheral Neuropathy (EORTC-QLQ-CIPN 20) questionnaire and ${ }^{99 \mathrm{~m}} \mathrm{Tc}$-labeled RBC scintigraphy were used to obtain patient data before treatment. During visits 2 to 11 (day 1 to 10), subjects underwent cyclic therapy. During visit 12 (day 11), the EORTC-QLQ-CIPN 20 and RBC scintigraphy were used again to obtain patient data after treatment (Table 1).

\section{Participants}

This study was an IIT. A total of 15 patients were recruited by the research manager after agreeing to interview the subjects (10 males and 5 females) in the outpatient clinic of each hospital Department of Hemato-oncology, based on specific criteria. The average age of the patients was $62.8 \pm 12.1$ years. Three of the 15 patients were diagnosed with colorectal cancer, while 12 others were diagnosed with stomach cancer, and the chemotherapy duration (CD) varied between patients. The vital signs of all the patients were stable.

The inclusion criteria were: 1 ) adults above 19 years of age; 2) patients diagnosed with stomach cancer, lung cancer, colon cancer, multiple myoma or lymphoma and treated with neurotoxic anti-cancer drugs such as oxalipatin, paclitaxel, docetaxel, thalidomide, vortexib, and vincristine; and 3) patients complaining of peripheral neuropathy (National Cancer Institute-Common Terminology Criteria for Adverse Events-class I and higher).

The exclusion criteria were: 1) sensory abnormalities due to central nervous system diseases or conditions such as stroke, cerebral hemorrhage, spinal cord compression syndrome, and spinal stenosis; 2) possible peripheral neuropathy associated with diabetes or alcohol-dependent disease; 3) treatment with neurotoxic drugs other than the anti-cancer drug mentioned in the selection criteria; 4) a diagnosis of peripheral vascular disease; 5) mental illness, which might prevent comprehension of the questionnaires and studies, due to limited or delayed intelligence or expression; 6) voluntary refusal of consent to participate in a clinical trial; 7) possible pregnancy or breastfeeding; 8) suppressed immunity; 9) critical condition; 10) vulnerable environment; and 11) drug addiction or alcoholism.

\section{Treatment}

In this study, all patients were treated with a device (healing-touch, HT-001; KAI Biotech, Jeonju, Korea) that generated warm and cool stimuli. The device consisted of thermoelectric elements capable of generating hot and cold energy according to the direction of current, a cooling system comprising a fan and heat sink, and a control system that set the target temperature, operating time, and the

Table 1. Observations and schedule

\begin{tabular}{lcccc}
\hline \multicolumn{1}{c}{ Contents } & Screening & Visit 1 & $\begin{array}{c}\text { Treatment } \\
\text { (Visit 2-11) }\end{array}$ & Visit 12 \\
\hline History & $\bullet$ & & & \\
Agreement & $\bullet$ & & & $\bullet$ \\
Vital sign & $\bullet$ & $\bullet$ & & $\bullet$ \\
Physical examination & $\bullet$ & $\bullet$ & & $\bullet$ \\
EORTC-QLQ-CIPN 20 & & $\bullet$ & & $\bullet$ \\
RBC scintigraphy & & $\bullet$ & & $\bullet$ \\
Subject number & $\bullet$ & & $\bullet$ \\
Abnormal reaction & & & $\bullet$ \\
Treatment & & & $\bullet$ \\
\hline
\end{tabular}

EORTC-QLQ-CIPN 20: European Organization for Research and Treatment of Cancer-Quality of Life Questionnaire-Chemotherapy-Induced Peripheral Neuropathy, RBC: red blood cell. 


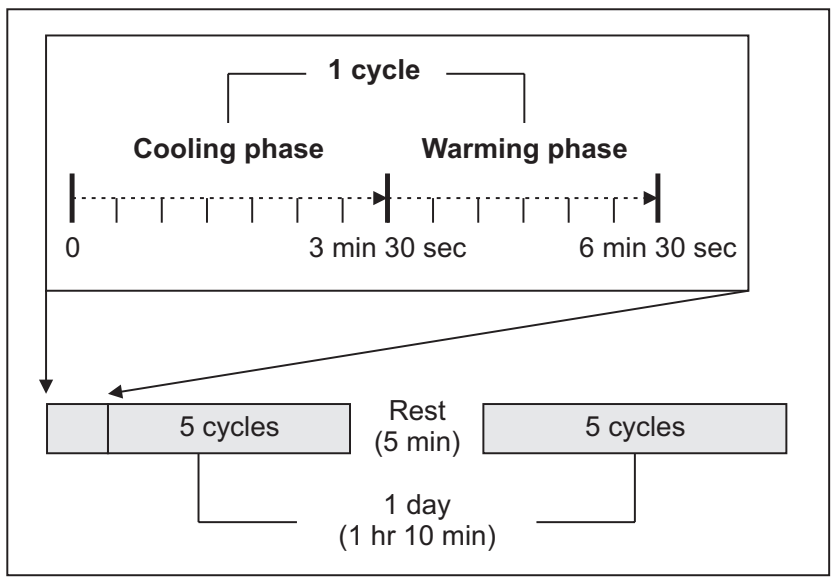

Fig. 1. Scheme of the treatment protocol. One cycle was lasted a total of 6 minutes and 30 seconds, including 3 minutes and 30 seconds of cooling phase and 3 minutes of warming phase. Total of 10 cycles was performed. Patients rested for 5 minutes after five cycles of treat. The treatment was performed for a total of 1 hour and 10 minutes at 1 day.

number of repetitions. The treatment protocol is shown in Fig. 1. All patients were treated with 10 cycles at each visit. Each cycle lasted a total of 6 minutes and 30 seconds, including 3 minutes and 30 seconds of cooling and 3 minutes of warming. The duration and temperature of stimulation were set according to the safety limits of the patients [20]. After five cycles of treatment, patients rested for 5 minutes. Treatment was performed for a total of 1 hour and $10 \mathrm{~min}$ utes at each visit. The target temperature of cooling was set at $15^{\circ} \mathrm{C}$ and the target temperature of warming was $41^{\circ} \mathrm{C}$. A thermal stimulation ranging between $15^{\circ}$ and $41^{\circ}$ was repeatedly delivered to the patient's hands.

\section{Assessment}

The subjects' blood volume was assessed via RBC scintigraphy before and after treatment in response to the EORTC-QLQ-CIPN 20 questionnaires.

\section{1) EORTC-QLQ-CIPN 20}

EORTC-QLQ-CIPN 20 questionnaires are used to measure the quality of life in patients with CIPN [21,22]. A total of 20 questions (Table 2) were included in each questionnaire and all patients responded before and after treatment. The total score, the score related to the upper limb, the scores closely related to the upper limb, and the scores unrelated to the upper limbs were calculated and used as a result.

\section{2) RBC scintigraphy}

RBC scintigraphy, a test procedure used in nuclear medicine, is designed for quantitative assessment of blood
Table 2. EORTC-QLQ-CIPN 20 questionnaires

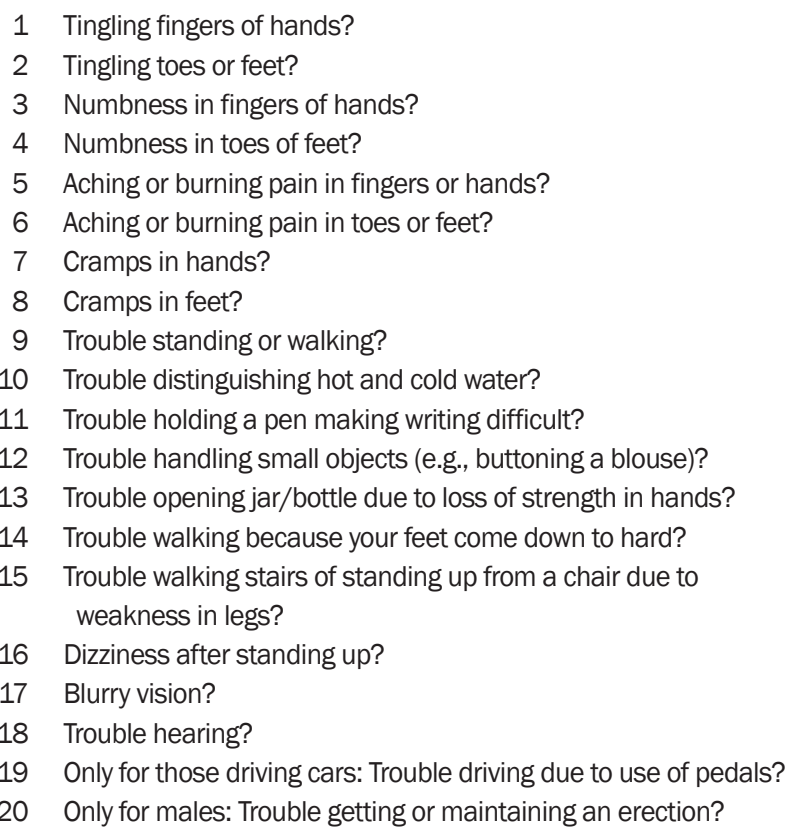

EORTC-QLQ-CIPN 20: European Organization for Research and Treatment of Cancer-Quality of Life Questionnaire-Chemotherapy-Induced Peripheral Neuropathy.

movement and blood volume by labeling RBC in the blood with the radioactive isotope ${ }^{99 \mathrm{~m}}$ Tc. First, the pyrophosphates are injected intravenously, and about 30 minutes later, $5 \mathrm{~mL}$ of the patient's blood is collected in a $10 \mathrm{~mL}$ syringe containing citrate-phosphate-dextrose-adenine $0.8 \mathrm{~mL}$ and $15 \mathrm{mCi}$ of ${ }^{99 \mathrm{~m}} \mathrm{TcO}_{4}$ and incubated at room temperature for about 10 minutes. After 5 to 10 minutes following injection of the incubated blood, the images at rest were acquired using a gamma camera for 2 minutes. After the rest image was acquired, the patient placed his or her hands on the device and the stress image was acquired using a gamma camera for 390 seconds. During the stress image acquisition, the device was operated under the same treatment cycle $\left(15^{\circ} \mathrm{C}\right.$ to $41^{\circ} \mathrm{C}, 390$ seconds). The gamma camera was set up to obtain the images at the rate of 1 frame per 3 seconds for a total of 40 resting images and 130 stress images. A set of rest images and three sets of stress images were obtained before and after treatment.

\section{Quantitative data analysis}

RBC scintigraphy was used for quantitative data analysis on a dedicated workstation PMOD 3.7 (PMOD Technologies, Zurich, Switzerland). When the gamma camera images were acquired, a standard source was used for each patient. The standard source was prepared with ${ }^{99 \mathrm{~m}} \mathrm{Tc}$ pertechnetate, which is the same radioisotope injected into the patients. The radioactivity of injection and the 

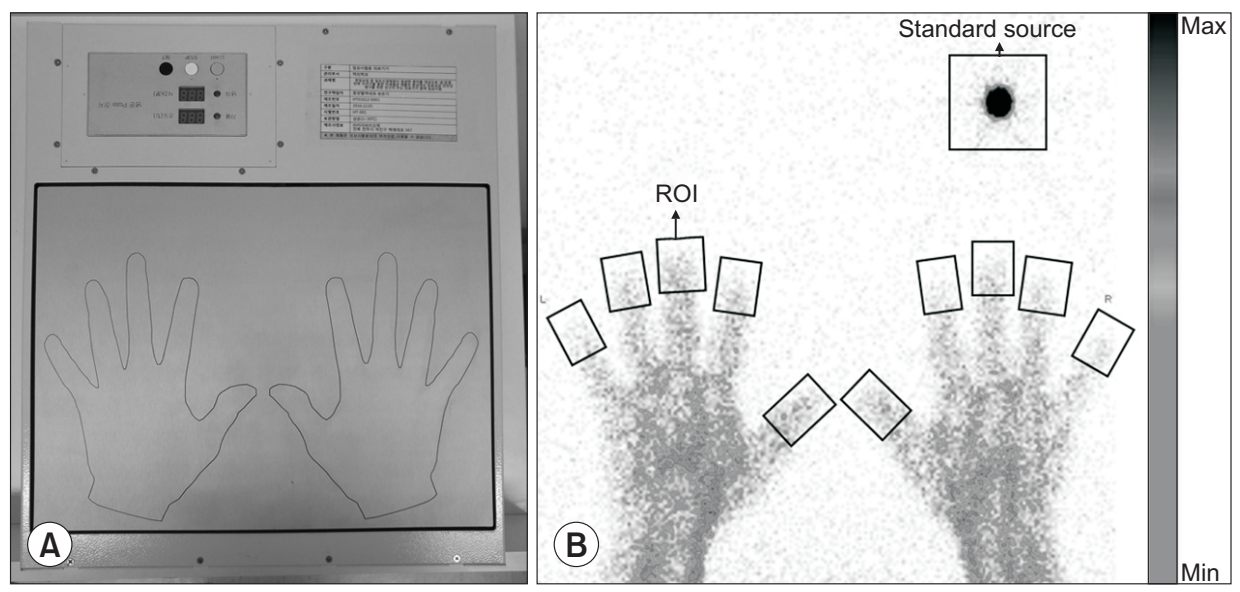

Fig. 2. Representative images of the method of treating the patient and red blood cell scintigraphy analysis. (A) Picture of the device. The patient puts his or her hands on the device. The hand position was printed on the device. (B) The standard source which is made by ${ }^{99 \mathrm{~m}} \mathrm{Tc}$ was located in the upper right side. The patients placed both hands on the cyclic therapy device. The ROls were drawn on each distal phalanx. ROI: region of interest, Min: minimum, Max: maximum.

standard source of ${ }^{99 \mathrm{~m}} \mathrm{Tc}$, injection time, and image acquisition times were recorded for decay correction during the quantitative analysis.

The RBC scintigraphic data consisted of 3 sets which were composed of 130 frames. Ten consecutive frames of data were selected as one data point; 13 data points were obtained for each set and a total of 39 data points were obtained. A region of interest (ROI) was drawn on each fingertip, and the ROI count was calculated. Fig. 2 presents the RBC image acquired and ROIs drawn on the fingertips and on the standard source. The quantitative analysis is represented by the following equation.

Radioactivity $(\mathrm{ROI})=\frac{\text { Radio activity }(\text { Standard source }) \times \text { Count value }(\text { ROI })}{\text { Count value }(\text { Standard source })}$

A simple comparison of ${ }^{99 \mathrm{~m}} \mathrm{Tc}$ uptake on the fingertip may not be accurate because the count or radioactivity of ${ }^{99 \mathrm{~m}} \mathrm{Tc}$ in each patient's fingertips differs according to the total injected dose. The ratio of fingertip radioactivity of the total ${ }^{99 \mathrm{~m}} \mathrm{Tc}$ injected dose was calculated and used as a result.

$$
\text { Blood volume (\%ID) }=\frac{\text { Fingertip radioactivity }}{\text { Total injected dose }} \times 100
$$

Because the blood volume at the fingertips in each hand represents a tiny fraction of the whole blood in a human, all comparisons were performed in the fingertips, and the blood volume results were expressed as common constants, $1 \times \mathrm{e}^{-3}$ in $\mathrm{T}$ and $1 \times \mathrm{e}^{-5}$ in $\mathrm{H}$.

\section{Statistical analysis}

The collected data were statistically analyzed using the IBM SPSS 25.0 program (IBM Co., Armonk, NY). The subjects' scores in response to the EORTC questionnaire, and blood volume measurements were calculated and expressed as mean \pm standard deviation. The collected data were subjected to statistical analysis via a paired $t$-test.
Table 3. Results of EORTC questionnaires score

\begin{tabular}{lc}
\hline \multicolumn{1}{c}{ Category } & $\begin{array}{c}\text { Mean } \pm \text { standard } \\
\text { deviation }\end{array}$ \\
\hline Total score & \\
$\quad$ Pre treatment & $41.6 \pm 12.8$ \\
Post treatment & $32.7 \pm 10.7$ \\
$P$ value & 0.002 \\
Score of related to upper limb questions & \\
Pre treatment & $17.7 \pm 6.4$ \\
Post treatment & $13.7 \pm 4.4$ \\
$P$ value & 0.002 \\
Score of closely related to upper limb questions & \\
Pre treatment & $8.6 \pm 3.2$ \\
Post treatment & $6.7 \pm 2.2$ \\
$P$ value & 0.006 \\
Score of excluding upper limb questions & \\
Pre treatment & \\
Post treatment & \\
$P$ value & $18.9 \pm 6.8$ \\
\hline
\end{tabular}

EORTC: European Organization for Research and Treatment of Cancer.

The relationship between the duration of chemotherapy and the treatment effects was subjected to correlation analysis (Spearman's rho). It was considered statistically significant when the $P$ value was below 0.05 .

\section{RESULTS}

\section{EORTC-QLQ-CIPN 20 questionnaire scores before and after treatment}

The results of the EORTC questionnaire were divided into a total score, scores related to upper limbs, score closely related to upper limbs, and scores excluding the upper limbs. The calculated scores are shown in Table 3.

The total score was $41.6 \pm 12.8$ before treatment and 32.7 \pm 10.7 after treatment $(P=0.002)$. The score related to up- 
Table 4. Results of change in blood flow before and after treatment by stimulation

\begin{tabular}{lcc}
\hline \multicolumn{1}{c}{ Category } & Mean \pm standard deviation & Variance \\
\hline Total & & \\
Pre treatment & $29.4 \pm 9.23 \mathrm{~T}$ & $8.52 \mathrm{H}$ \\
Post treatment & $25.8 \pm 7.89 \mathrm{~T}$ & $6.23 \mathrm{H}$ \\
$P$ value & $<0.001$ & $<0.001$ \\
Cooling phase & & \\
Pre treatment & $29.6 \pm 9.38 \mathrm{~T}$ & $8.80 \mathrm{H}$ \\
Post treatment & $26.0 \pm 8.05 \mathrm{~T}$ & $6.48 \mathrm{H}$ \\
$P$ value & $<0.001$ & $<0.001$ \\
Warming phase & & \\
Pre treatment & $28.7 \pm 9.17 \mathrm{~T}$ & $8.41 \mathrm{H}$ \\
Post treatment & $25.2 \pm 7.50 \mathrm{~T}$ & $5.63 \mathrm{H}$ \\
$P$ value & $<0.001$ & $<0.001$ \\
\hline
\end{tabular}

T: $1.0 \times \mathrm{e}^{-3}, \mathrm{H}: 1.0 \times \mathrm{e}^{-5}$.

per limb questions was $17.7 \pm 6.4$ before and $13.7 \pm 4.4$ after treatment $(P=0.002)$. The score closely related to upper limb questions was $8.6 \pm 3.2$ before and $6.7 \pm 2.2$ after treatment $(P=0.006)$. The score excluding upper limb questions was $23.9 \pm 7.0$ before and $18.9 \pm 6.8$ after treatment $(P$ $=0.002)$.

\section{Blood volume before and after treatment}

The blood volume determined via quantitative analysis is shown in Table 4. Total blood volume is made up of the results that contain the data obtained from rest and stress periods of RBC scintigraphy. Before treatment, the total blood volume was $29.4 \pm 9.23 \mathrm{~T}$. After treatment, the total blood volume was $25.8 \pm 7.89 \mathrm{~T}(P<0.001)$. The variance in blood volume was $8.52 \mathrm{H}$ before treatment and $6.23 \mathrm{H}$ after treatment $(P<0.001)$.

\section{The change in blood volume before and after treatment by stimulation}

Blood volume before and after exposure to cool or warm stimuli were identified (Table 4). During the cooling phase, the blood volume was $29.6 \pm 9.38 \mathrm{~T}$ before and $26.0 \pm 8.05$ T after treatment $(P<0.001)$, and the variance of blood volume was $8.80 \mathrm{H}$ before and $6.48 \mathrm{H}$ after treatment $(P<$ $0.001)$. During the warming phase, the blood volume was $28.7 \pm 9.17 \mathrm{~T}$ before and $25.2 \pm 7.50 \mathrm{~T}$ after treatment $(P<$ 0.001 ), and the variance of blood volume was $8.41 \mathrm{H}$ before and $5.63 \mathrm{H}$ after treatment $(P<0.001)$.

\section{Correlation between the duration of chemotherapy and the effect of treatment}

The correlation between CD and treatment effect was analyzed. The CD was set from the start of chemotherapy to the start of treatment via cycles of cooling and warming therapy. The results of correlation analysis of the average blood-volume difference of each patient ( $\Delta$ Average), the variance difference of average blood volume ( $\Delta$ Variance), the EORTC-QLQ-CIPN20 questionnaires score difference ( $\Delta$ Score), and the CD are presented in Table 5. The correlation coefficient between $\mathrm{CD}$ and $\Delta$ Score was $-0.599(P=$ $0.042)$.

\section{DISCUSSION}

Currently, CIPN patients are treated via pharmacological and non-pharmacological approaches; however, it is not based on any theoretical background, because of the paucity of studies involving cancer patients $[6,7]$. This clinical study evaluated the usefulness of cyclic thermal therapy for patients with CIPN. The advantages of cyclic thermal therapy may be mediated via induction of vasoconstriction and vasodilation, decreased edema, and altered blood flow [23]. We investigated the relationship between symptom relief and blood volume at the fingertips calculated quantitatively using RBC scintigraphy. RBC scintigraphy is appropriate for the measurement of blood flow and blood flow changes occurring in peripheral tissue compared to other diagnostic methods.

In this study, 15 CIPN patients were exposed to cyclic thermal therapy for a total of 10 times at each visit to ameliorate CIPN symptoms via possible improvement in blood circulation. To monitor the changes in CIPN symptoms, the EORTC-QLQ-CIPN 20 was administered before and after treatment, and RBC scintigraphy was performed before and after treatment to determine the changes in blood volume. Although the number of participants was 15, there were 6 independent data sets from the cooling and warming phase in pre- and post-treatment for each patient, and each data set was composed of numerous resultant corresponding values for each of the acquisition time points. It was judged that sufficient comparison would be possible with the data set.

In general, the items in the EORTC-QLQ-CIPN 20, which is used to diagnose CIPN, were divided into three types: sensory symptoms and defects, motor, and autonomic scales [24]. In this study, the items listed in the questionnaires were used to calculate the total scores, the scores related to the upper limbs, the scores closely related to the upper limbs, and the scores excluding the upper limbs, because the patients' upper limbs were treated directly.

The total questionnaire score was reduced from $41.6 \pm$ 12.8 before treatment to $32.7 \pm 10.7$ points after treatment $(P=0.002)$. The scores related to upper limbs (question 
Table 5. Amount of change in patient's data and results of correlation analysis

\begin{tabular}{|c|c|c|c|c|c|}
\hline No. & $\Delta$ Average & \multicolumn{2}{|c|}{$\Delta$ Variance } & $\Delta$ Score & CD (day) \\
\hline 1 & 0.00588411 & \multicolumn{2}{|c|}{0.00000002} & -23 & 321 \\
\hline 2 & -0.00824121 & \multicolumn{2}{|c|}{-0.00000441} & -3 & 113 \\
\hline 3 & 0.00112180 & \multicolumn{2}{|c|}{-0.00000054} & -32 & 3,697 \\
\hline 4 & -0.00857883 & \multicolumn{2}{|c|}{-0.00004514} & -2 & 180 \\
\hline 5 & -0.01130238 & \multicolumn{2}{|c|}{-0.00000693} & -3 & 186 \\
\hline 6 & -0.00230905 & \multicolumn{2}{|c|}{-0.00000005} & -8 & 504 \\
\hline 7 & 0.00004785 & \multicolumn{2}{|c|}{0.00000316} & -9 & 226 \\
\hline 8 & -0.00729858 & \multicolumn{2}{|c|}{-0.00000006} & -17 & 336 \\
\hline 9 & -0.00331466 & \multicolumn{2}{|c|}{0.00000240} & -7 & 441 \\
\hline 10 & -0.00826953 & \multicolumn{2}{|c|}{-0.00000271} & 4 & 256 \\
\hline 11 & -0.00393023 & \multicolumn{2}{|c|}{-0.00001155} & -8 & 154 \\
\hline 12 & 0.00078119 & \multicolumn{2}{|c|}{-0.00000317} & 0 & 84 \\
\hline 13 & -0.00146187 & \multicolumn{2}{|c|}{-0.00000553} & -14 & 301 \\
\hline 14 & 0.00731170 & \multicolumn{2}{|c|}{0.00001190} & 1 & 21 \\
\hline \multirow[t]{2}{*}{15} & -0.01399654 & \multicolumn{2}{|c|}{-0.00000367} & -13 & 144 \\
\hline & & & $\Delta$ Average & $\Delta$ Variance & $\Delta$ Score \\
\hline \multirow[t]{3}{*}{ Spearman's rho } & \multirow[t]{3}{*}{$\mathrm{CD}$} & Correlation coefficient & 0.143 & 0.282 & $-0.599^{a}$ \\
\hline & & Sig. (2-tailed) & 0.612 & 0.308 & 0.018 \\
\hline & & $\mathrm{N}$ & 15 & 15 & 15 \\
\hline
\end{tabular}

$\mathrm{CD}$ : chemotherapy duration.

${ }^{\mathrm{a}} P=0.042$.

numbers $1,3,5,7,10,11,12$, and 13) were reduced from $17.7 \pm 6.4$ before treatment to $13.7 \pm 4.4$ after treatment $(P=$ 0.002 ). The scores closely related to the upper limbs (question numbers $1,3,5$, and 7 ) were reduced form $8.6 \pm 3.2$ before treatment to $6.7 \pm 2.2$ after treatment $(P=0.006)$. The results also showed that the scores excluding upper limbs were reduced from $23.9 \pm 7.0$ to $18.9 \pm 6.8(P=0.002)$. The results suggest a possible attenuation of CIPN symptoms involving the upper limbs by improving the blood circulation in both hands during the cyclic thermal therapy. In addition, interestingly, the scores excluding the upper limbs were also reduced after treatment. The results, in conjunction with the results from the upper extremities, suggest that circulation was improved in the vessels of the peripheral tissues following cyclic therapy, and this effect was widespread throughout the body. Finally, in patients with CIPN, the whole-body symptoms were mitigated.

In this study, a contact thermal stimulation device was used to provide cyclical thermal therapy. Several types of drugs, including pain killers, are being used to treat symptoms such as pain and edema in CIPN patients. However, because drug treatment alone does not have much effect on patients' symptoms, it is accompanied by thermal physical therapy which is uses cold or hot packs. An alternative hydrotherapy, infrequently used in clinics, is a method of dipping the body in tanks containing cold and hot water. But in this study, we applied a cyclic thermal therapy that periodically and repeatedly provided hot and cold temperatures during a relatively short period.

As a preclinical study, we had already confirmed that this alternative cyclic thermal therapy has the effect of inflammatory inhibition, swelling reduction, and vascular permeability reduction in a trimellitic anhydride-induced acute contact hypersensitivity mouse model [25]. These results are thought to be one of the mechanisms for interpreting clinical results from CIPN patients, and one of the appropriate ways to prove them is RBC scintigraphy which can be evaluated by quantification of blood flow volumes.

RBC scintigraphy was used to assess the blood volume at rest and the changes in blood volume, reflected by stress in any parts of body, including both hands, and quantitative analysis in the ROI, here in the fingertips. RBC scintigraphy can provide objective, repetitive, and quantitative information compared to other modalities in the peripheral tissues. The fingertip area was selected for analysis due to the abundant microvascular and nerve distribution in this area. Most patients' symptoms involve the microvascular and nervous systems, whereas large vessels act as mere conduits for blood.

The results of RBC scintigraphy suggested that the blood volume was reduced from $29.4 \pm 9.23 \mathrm{~T}$ before to $25.8 \pm$ $7.89 \mathrm{~T}$ after treatment $(P<0.001)$. Based on stress type, the blood volume in the cooling phase was decreased from $29.6 \pm 9.38 \mathrm{~T}$ before treatment to $26.0 \pm 8.05 \mathrm{~T}$ after treatment $(P<0.001)$. Also, it was decreased from $28.7 \pm 9.17 \mathrm{~T}$ before treatment to $25.2 \pm 7.50 \mathrm{~T}$ after treatment during the 
warming phase $(P<0.001)$.

The blood volume and blood volume variance in patients was decreased following cyclic thermal therapy due to effective circulation and the reduction of interstitial edema via gradual and consecutive vasoconstriction and vasodilation of peripheral blood vessels. Most CIPN patients, even with varying severity, have swollen hands, weak hands, and numbness. Vascular damage occurs due to chemotherapy, and the resulting increase in capillary permeability triggers edema [26], and peripheral blood stasis, which may interfere with effective circulation. The decrease in blood volume after cyclic thermal therapy is attributed to a reduction in edema and blood stasis following restoration of the vascular reactivity of damaged blood vessels. The circulation may have increased via a reduction of blood stasis, which resulted in the restoration of micro-circulation in the whole body as well as both upper extremities, thereby mitigating the symptoms of CIPN.

Of all the variations in blood volume during the whole study period, the blood volume in the cooling and warming phases was significantly decreased after treatment compared with pre-treatment values, as shown in Table 4 . The results show that vascular constriction and relaxation, upon exposure to the stimuli, were stabilized, thereby maintaining the reactivity of the vessels. Vascular reactivity can be said to be a stable response of blood vessels to causes of change, and maintaining vascular reactivity means that the organs or peripheral tissues of our body are stable against external changes. Therefore, it can be interpreted that blood flow is recovering from vascular reactivity that does not show much difference in each of the periods after treatment.

The relationship between $\mathrm{CD}$ and symptom improvement is presented in Table 5. The CD was not related to changes in average blood volume and variance but was correlated with changes in the total scores of the questionnaire. These results demonstrate an uplifting effect on patients under prolonged chemotherapy and may be related to psychological support associated with cyclic thermal therapy as well as objective changes in circulation. The results also suggest that chemotherapy induces peripheral microangiopathy in the peripheral tissue as well as peripheral neural injury.

There has been no study qualitatively evaluating the blood volume in the peripheral tissue and fingertips. In this study, we introduced RBC scintigraphy to evaluate blood volume and its change by cyclic thermal stimulation, and to demonstrate vascular instability and validate a therapeutic effect on subjective symptoms and objective changes in vascular reactivity. In our other study using cyclic thermal stimulation RBC scintigraphy for stroke patients, we found that peripheral vasoreactivity and neuropathy were related [27]. Cyclic thermal stimulation could show the status of peripheral vasoreactivity during about 30 minutes of stimulation. In this study, we applied cyclic thermal therapy repetitively for 10 days to patients with CIPN and observed changes in vasoreactivity and symptoms. We tried to see whether repeated application of this stimulation could have a therapeutic effect. After analyzing the results of the RBC scintigraphy and EORTCQLQ-CIPN 20 scores, we confirmed that vasoreactivity was strengthened and EORTC-QLQ-CIPN 20 scores were lowered after applying cyclic thermal therapy. When the studies were combined, the CIPN symptoms were alleviated by increasing vasoreactivity using cyclic thermal therapy, which means that cyclic thermal therapy can be used as a treatment for CIPN.

The limitations of this study are that when cyclic thermal therapy was applied to patients with CIPN, it was not compared with the results of applying cold or warm alone, and that the number of participants was set at 15 based on a calculation of sample size in an investigator-initiated trial. Because the pre- and post-treatment RBC scintigraphic quantitative data created 39 data point for each patient, we could make a sufficient comparison. Most patients suffered a lot from chemotherapy before participating in this study, and could not use the existing method because they had had experienced no particular effect from existing treatments (include thermal therapy). The research before and after the application of this treatment will confirm its effectiveness. Second, there is a limit in not being able to work with other methods besides RBC scintigraphy to measure peripheral vasoreactivity. In addition to the results of the survey on neuropathy treatment, except for the questionnaire result, further study is needed on neurological tests. Further research using this treatment in the clinical setting is planned. Third, in this study, no research has been done on how long the therapeutic effects last. Further research on the relationship between treatment duration and treatment effectiveness needs to be continued. Since this study was the first research of its kind to be attempted, it was arbitrarily set up as 10 times for 2 weeks considering the ease of application and the duration of treatment effects for patients.

In future studies, we will apply RBC scintigraphy to conduct a comparative study of each of the therapeutic tools. Also, although it is difficult to conduct studies with patients, it is necessary to investigate additional pathophysiological mechanisms underlying chemotherapy-induced neuropathy and microangiopathy at least in animals. Further studies including the use of different evaluation tools are needed to address these limitations.

In conclusion, patients diagnosed with CIPN were surveyed using questionnaires and $\mathrm{RBC}$ scintigraphy to in- 
vestigate the severity of CIPN-causing symptoms and peripheral blood volume before and after exposure to cyclic thermal therapy. The CIPN symptoms and blood stasis, possibly brought about by chemotherapy-induced edema, were improved, and the vascular reactivity was stabilized. Therefore, we suggest that cyclic thermal therapy is useful in ameliorating CIPN symptoms by improving blood circulation. Additionally, RBC scintigraphy can be used to objectively evaluate blood volume under specific stress conditions as well as at rest in peripheral tissues. It is expected that the application of RBC scintigraphy can be expanded by utilizing blood volume information to visualize and objectively analyze the vascular response to stimuli.

\section{CONFLICT OF INTEREST}

No potential conflict of interest relevant to this article was reported.

\section{FUNDING}

This research was supported by a grant of the Korea Health Technology R\&D Project through the Korea Health Industry Development Institute (KHIDI), funded by the Ministry of Health \& Welfare, Republic of Korea (grant number: HI15C1529) and the National Research Foundation of Korea (NRF) grant funded by the Korea government (MSIT) (2020R1F1A1050711).

\section{ORCID}

Minjoo Kim, https://orcid.org/0000-0002-5435-3974 Eun-Mi Kim, https://orcid.org/0000-0001-6222-3486 Phil-Sun Oh, https://orcid.org/0000-0002-0711-7514 Seok Tae Lim, https://orcid.org/0000-0002-3407-7212 Myung-Hee Sohn, https://orcid.org/0000-0002-7955-721X Eun-Kee Song, https://orcid.org/0000-0001-8836-0294 Keon Uk Park, https://orcid.org/0000-0002-6902-7063 Jin Young Kim, https://orcid.org/0000-0002-9597-9834 Kyoung Sook Won, https://orcid.org/0000-0001-7185-911X Hwan-Jeong Jeong, https://orcid.org/0000-0002-9539-342X

\section{REFERENCES}

1. Windebank AJ, Grisold W. Chemotherapy-induced neuropathy. J Peripher Nerv Syst 2008; 13: 27-46.

2. Armstrong T, Almadrones L, Gilbert MR. Chemotherapyinduced peripheral neuropathy. Oncol Nurs Forum 2005; 32:
305-11.

3. Kumar SP, Sisodia V. Chemotherapy-induced or chemotherapy-associated? Does physical therapy play a role in prevention and/or management of peripheral neurotoxicity and neuropathy? Indian J Palliat Care 2013; 19: 77-8.

4. Wolf S, Barton D, Kottschade L, Grothey A, Loprinzi C. Chemotherapy-induced peripheral neuropathy: prevention and treatment strategies. Eur J Cancer 2008; 44: 1507-15.

5 . Woo IS. Recent updates on chemotherapy-induced peripheral neuropathy. Korean J Med 2015; 88: 35-7.

6. Barton DL, Wos EJ, Qin R, Mattar BI, Green NB, Lanier KS, et al. A double-blind, placebo-controlled trial of a topical treatment for chemotherapy-induced peripheral neuropathy: NCCTG trial N06CA. Support Care Cancer 2011; 19: 833-41.

7. Wickham R. Chemotherapy-induced peripheral neuropathy: a review and implications for oncology nursing practice. Clin J Oncol Nurs 2007; 11: 361-76.

8. Sutterfield SL, Caldwell JT, Post HK, Lovoy GM, Banister HR, Ade CJ. Lower cutaneous microvascular reactivity in adult cancer patients receiving chemotherapy. J Appl Physiol (1985) 2018; 125: 1141-9.

9. Akasaki Y, Miyata M, Eto H, Shirasawa T, Hamada N, Ikeda $\mathrm{Y}$, et al. Repeated thermal therapy up-regulates endothelial nitric oxide synthase and augments angiogenesis in a mouse model of hindlimb ischemia. Circ J 2006; 70: 463-70.

10. Tepperman PS, Devlin M. The therapeutic use of local heat and cold. Can Fam Physician 1986; 32: 1110-4.

11. Johnson JM, Minson CT, Kellogg DL Jr. Cutaneous vasodilator and vasoconstrictor mechanisms in temperature regulation. Compr Physiol 2014; 4: 33-89.

12. Maeda H, Kurose T, Nosaka S, Kawamata S. Microvascular circulation at cool, normal and warm temperatures in rat leg muscles examined by histochemistry using Lycopersicon esculentum lectin. Acta Histochem 2014; 116: 1096-103.

13. Salmi AM, Hong C, Futrell JW. Preoperative cooling and warming of the donor site increase survival of skin flaps by the mechanism of ischaemic preconditioning: an experimental study in rats. Scand J Plast Reconstr Surg Hand Surg 1999; 33: 163-7.

14. Roque FR, Hernanz R, Salaices M, Briones AM. Exercise training and cardiometabolic diseases: focus on the vascular system. Curr Hypertens Rep 2013; 15: 204-14.

15. Bang SH, Oh YS, Park HJ, Lee TK, Yang JS, Lee SM, et al. Evaluation of finger blood flow with Tc-99m MDP (methylene diphosphonate). Korean J Intern Med 1992; 7: 94-101.

16. Kunnen JJ, Dahler HP, Doorenspleet JG, van Oene JC. Effects of intra-arterial ketanserin in Raynaud's phenomenon assessed by 99MTc-pertechnetate scintigraphy. Eur J Clin Pharmacol 1988; 34: 267-71.

17. Sarikaya A, Ege T, Firat MF, Duran E. Assessment of digital ischaemia and evaluation of response to therapy by $99 \mathrm{mTc}$ sestamibi limb scintigraphy after local cooling of the hands 
in patients with vasospastic Raynaud's syndrome. Nucl Med Commun 2004; 25: 207-11.

18. Kaminaga T, Takada K, Kutomi K, Imai E, Furui S. Improvement in cold-induced Raynaud's syndrome with administration of ticlopidine hydrochloride detected by Tc-99m DTPA human serum albumin scintigraphy. Clin Nucl Med 2002; 27: 131-2.

19. Garai I, Csiki Z, Galuska L. Is MIBI the best choice for the evaluation of hand perfusion during cold test provocation? Nucl Med Commun 2005; 26: 269-70.

20. Habash RW, Bansal R, Krewski D, Alhafid HT. Thermal therapy, part 1: an introduction to thermal therapy. Crit Rev Biomed Eng 2006; 34: 459-89.

21. Postma TJ, Aaronson NK, Heimans JJ, Muller MJ, Hildebrand JG, Delattre JY, et al. The development of an EORTC quality of life questionnaire to assess chemotherapy-induced peripheral neuropathy: the QLQ-CIPN20. Eur J Cancer 2005; 41: 1135-9.

22. Wolf SL, Barton DL, Qin R, Wos EJ, Sloan JA, Liu H, et al. The relationship between numbness, tingling, and shooting/ burning pain in patients with chemotherapy-induced pe- ripheral neuropathy (CIPN) as measured by the EORTC QLQCIPN20 instrument, N06CA. Support Care Cancer 2012; 20: 625-32.

23. Hing WA, White SG, Bouaaphone A, Lee P. Contrast therapy-a systematic review. Phys Ther Sport 2008; 9: 148-61.

24. Mols F, van de Poll-Franse LV, Vreugdenhil G, Beijers AJ, Kieffer JM, Aaronson NK, et al. Reference data of the European Organisation for Research and Treatment of Cancer (EORTC) QLQ-CIPN20 Questionnaire in the general Dutch population. Eur J Cancer 2016; 69: 28-38.

25. Piao CH, Fan YJ, Nguyen TV, Song CH, Jeong HJ, Chai OH. Effects of thermal therapy combined with blue light-emitting diode irradiation on trimellitic anhydride-induced acute contact hypersensitivity mouse model. J Dermatolog Treat 2020. doi: 10.1080/09546634.2020.1809622.

26. Diskin CJ, Stokes TJ, Dansby LM, Carter TB, Radcliff L, Thomas SG. Towards an understanding of oedema. BMJ 1999; 318: 1610-3.

27. Wang SJ, Lee CH, Kang HG, Kim KW, Kim M, Jeong HJ, et al. Peripheral vasoreactivity in acute ischemic stroke with hemiplegia. Sci Rep 2021; 11: 8531. 